\title{
MEDIDA INDIRETA DA PRESSÃO ARTERIAL EM FUNÇÃO DA LARGURA DO MANGUITO, EM PACIENTES NAS FASES PRÉ- OPERATORIA IMEDIATA, RECEPÇÃO NO CENTRO CIRURGICO E POS ANESTÉSICA
}

\author{
Aparecida de Cassia Giani Peniche* \\ Edna Apparecida Moura Arcuri**
}

PENICHE, A. de C.G.; ARCURI, E.A.M. Medida indireta da pressão arterial em função da largura do manguito, em pacientes nas fases pré-operatória imediata recepção no centro cirúrgico e pós anestésica. Rev. Esc. Enf. USP, v. 26, n. 2, p. 243-56, Ago. 1992.

Este estudo visou comparar em pacientes submetidos a cirurgias, medidas de pressão arterial obtidas com duas larguras de manguito: a largura padrão, de $12 \mathrm{~cm}$, usada internacionalmente, e a largura correspondente a 0,38 da medida da circunferência do braço, recomendada pela American Heart Association, para evitar hiper ou hipoestimação nos valores obtidos por ocasião do registro da pressão arterial. As comparações foram efetuadas nas fases pré-operatória imediata, recepção no centro cirúrgico e pós anestésica, tendo em vista, a importância de se detectar alterações com o máximo de precisão, num periodo de avaliação geral das condiçōes impostas pelo ato cirúrgico. Os resultados obtidos confirmam diferenças significantes $(p<0,0001)$ entre as duas larguras de manguitos utilizados, atingindo em alguns individuos $30 \mathrm{mmHg}$ hipoestimação nos valores sistólicos e diastólicos.

UNITERMOS: Determinaçĩo da pressão arterial. Pressão arterial.

\section{INTRODUÇÃO}

A dor, hipotermia, posicionamento incorreto, hipoventilação, retenção urinária e outros fatores decorrentes do ato anestésico-cirúrgico, associados entre si, devem ser considerados de relevada importância, assim como as alterações do sistema cardiovascular, o qual responde continuamente ao estresse e alterações provocadas pelo ato cirúrgico. A resposta a essas alterações é dada através de mecanismos nervosos e humorais, que atuam rapidamente na regulação da pressão arterial, associados sobretudo à função renal e ao volume sanguiíneo.

A pressão arterial, produto do débito cardíaco e da resistência periférica, é portanto o elemento fundamental da dinâmica sangüínea

\footnotetext{
* Assistente do Departamento de Enfermagem Médico-Cirúrgica da Escola de Enfermagem USP.

** Prof. Titular do Departamento de Enfermagem Médico-Cirúrgica da EEUSP.
} 
e fator preponderante na manutenção do aporte de oxigênio a todos os tecidos do organismo.

O estresse emocional e a ansiedade que acompanham a fase préoperatória e a vulnerabilidade do organismo diante dos diversos fatores estressantes durante $o$ ato cirúrgico, como as drogas e o sangramento, resultam em alterações da pressão arterial que necessitam ser imediatamente detectadas, no sentido de garantir continuidade da oferta de oxigênio diante das demandas de cada momento.

Alguns autores como CULLEN (1977), DRAIN; SHIPLEY (1981), FERRAZ (1980), NOCITE (1987), têm enfatizado a responsabilidade dos elementos da equipe de enfermagem prevenirem riscos, detectando precocemente complicações respiratórias e cardiovasculares, oferecendo assistência individualizada ao paciente.

Com o desenvolvimento das novas técnicas cirúrgicas e anestésicas, maior número de intervenções foi possível, com variada duração. Consequientemente aumentaram os problemas inerentes às técnicas cirúrgicas e à transição entre o estado de anestesia e o retorno à homeostase e consciência (NOCITE, 1987).

Os fatos relatados evidenciaram a necessidade de um local que centralizasse os pacientes após o término da cirurgia, com o objetivo de dar condições de restabelecer suas funções fisiológicas e a consciência, sob a observação continuada e atuação de uma equipe de enfermagem especializada, atuando organizada e sistematicamente a fim de detectar rapidamente alterações na evolução clínica do paciente (JOUCLAS, 1987). Surgiu então a Sala de Recuperação Anestésica (SRA), local onde os pacientes permanecem durante um período após o ato cirúrgico. CONSEILLER et al (1975) enfatizaram como prioridade na SRA a manutenção das vias aéreas, a adequada ventilação pulmonar do paciente e a manutenção da pressão arterial.

As alterações da pressão arterial não só em SRA são detectadas pela medida desse parâmetro, comumente realizada por método in. direto (auscultatório), cuja vantagem principal é não ser invasivo e poder ser correlacionado com as medidas indiretas, já registradas no período perioperatório e por ocasião da anamnese do paciente (ALDRETE; KROULIK, 1970).

O método, indireto, porém, vem recebendo críticas por ser um método impreciso, sendo que a medida da pressão arterial já constituía preocupação no inicío do século, e ao final da década de 30 , especialistas americanos e ingleses reuniam-se num comitê para estudar sua padronização (AMERICAN HEART ASSOCIATION AND CARDIAC SOCIETY OF GREAT BRITAIN AND IRELAND, 1939).

Identificam-se na literatura, diversas publicações que discutem as variáveis mais implicadas na imprecisão do método auscultatório (WILCOX, 1961; ROSE et al, 1966; KIRKENDALL et al, 1980; KARVONEN et al, 1964; MANCIA et al, 1987), as quais permitem concluir, que considerável fração do erro da medida pode ser controlada. Na 
análise desses estudos realizados em 1939 até os dias atuais, identificou-se como fator crucial no registro indireto da pressão arterial, a influência causada pela largura do manguito (bolsa inflável de borracha) do esfigmomanômetro.

Testando a recomendação da AMERICAN HEART ASSOCIATION, no que diz respeito à influência da largura do manguito na medida da pressão arterial, ARCURI (1988) constatou que o manguito de largura padrão, (MLP, que é o manguito com $12 \mathrm{~cm}$ de largura usado internacionalmente) causa importante hipoestimação da pressão ar. terial em pessoa cujas circunferências de braço são menores que $28 \mathrm{~cm}$. Essa hipoestimação, entretanto, difere entre pessoas com braços de circunferências semelhantes e pode ser muito aumentada em braços com circunferências menores do que a citada.

No tocante à importância de se detectar com precisão uma hipo ou hipertensão, comuns no período pós operatório, e sabendo que alterações da pressão arterial podem ser um sinal de situações graves, (CULLEN, 1977; POUND, 1987; WEBB, 1977) como a hemorragia interna, pensou-se em iniciar estudos que pudessem resultar em melhor confiabilidade no uso do método indireto em pacientes cirúr. gicos.

\section{OBJETIVOS}

Estudar os valores da pressão arterial de um grupo de pacientes submetidos ao ato cirúrgico identificando e analisando:

- As larguras dos manguitos mais utilizados na população de estudo.

- As médias da pressão arterial registradas pelo manguito de largura correta (MLC) e pelo manguito de largura padrão (MLP) nas fases: pré-operatória imediata, de recepção no cen. tro cirúrgico e pós-anestésica.

\section{METODOLOGIA}

\section{1 - População}

A amostra populacional foi composta de 101 pacientes internados em um hospital geral governamental de ensino, com a finalidade de serem submetidos a intervenção cirúrgica.

\section{2 - Critérios para seleção da amostra}

Foram incluídos na amostra os pacientes que atenderam os seguintes requisitos:

- faixa etária de 16-60 anos;

- pertencentes ao programa de cirurgias eletivas; 
- que fossem encaminhados à sala de recuperação anestésica ao final do ato anestésico-cirúrgico;

- que apresentassem respiração espontânea no período pós anestésico;

- que tivessem o membro superior esquerdo livre de restriçōes para as medidas de pressão arterial;

- que tivessem a circunferência do braço maior que $32 \mathrm{~cm}$ e menor que $30 \mathrm{~cm}$.

\section{3 - Coleta de dados}

A medida indireta da pressão arterial foi realizada com base no protocolo preconizado por ARCURI (1985), e adaptado para ser realizado em 3 fases distintas a saber:

Fase pré-operatória imediata. Compreendida desde a véspera da cirurgia até o momento em que o paciente é recebido no centro cirúrgico. (CASTELLANOS; JOUCLAS, 1990). Nesse períođo a medida da pressão arterial foi realizada na unidade de internação (clínica cirúrgica).

Fase de recepção do paciente no centro cirúrgico. A medida da pressão arterial foi realizada em área física denominada recepção do paciente onde ele permaneceu aproximadamente 30 minutos, até ser conduzido à sala de operações.

Fase pós anestésica. Medidas realizadas durante a permanência na SRA. A primeira medida era feita no momento da chegada do paciente nesta sala (tempo zero). Em seguida, eram efetuadas medidas a cada 30 minutos, até completar 3 horas de permanência na SRA.

Cabe ressaltar as razões de se medir a pressão arterial em três fases distintas, uma vez que o objetivo desse estudo não foi a comparação entre as fases, e sim a comparação entre as medidas feitas com manguitos de larguras diferentes em cada fase. Convém salientar, que a equipe pertencente à SRA onde ocorreu a coleta de dados se utiliza do índice de ALDRETE; KROULIK, 1970 para avaliação do desempenho fisiológico dos pacientes. Esse índice estabelece, que para o paciente ter seus sistemas avaliados e obter alta da SRA, além do retorno da consciência e reflexos, a pressão arterial em SRA deverá estar compreendida no mínimo em $80 \%$ da pressão obtida no préoperatório. Surge dessa realidade, a necessidade de se obter a pressão arterial nas fases pré-operatória imediata e recepção.

Considerando, entretanto, que a permanência na SRA é justificada até que seja atingida a estabilidade dos sinais vittais, o retorno da consciência e reflexos presentes (ALDRETE; KROULIK, 1970; CUNHA et al, 1977; POSSO, 1975), alguns pacientes recebiam alta antes de completar 3 horas de permanência nessa sala. Neles a pressão arterial foi medida até o momento da alta. 


\subsection{Recursos humanos-treinamento}

Foi desenvolvida a técnica utilizada no laboratório de Esfigmomanometria do Departamento Médico-Cirúrgico da Escola de Enfermagem da Universidade de São Paulo e utilizado o estetoscópio duplo que tem como finalidade eliminar erros, de preferência pelo dígito final, oferecer segurança na definição das pressões sistólica e diastólica, controlar a velocidade de inflação e deflação (AMERICAN HEART ASSOCIATION, 1980; BAZET et al, 1933; POMIDOSSI, et al, 1985; WILCOX, 1961) e eliminar erros decorrentes de posições incorretas e de instrumentação, assim como diminuir o alerta do cliente.

\subsection{Material utilizado}

- 1 estetoscópio Tycos simples.

- 2 estigmomanômetros aneróides Tycos testados semanalmente contra um esfigmomanômetro de mercúrio, por meio de um tubo de borracha em $\mathbf{Y}$.

- 7 manguitos, cujas larguras das bolsas de borracha variaram de $1 \mathrm{em} 1 \mathrm{~cm}(8,9,10,11,12,13$ e $14 \mathrm{~cm})$. O comprimento de cada bolsa foi estabelecido no sentido de manter a mesma proporção largura/comprimento do manguito de largura padrão, aproximadamente 1:2. Assim, todos os manguitos de largura correta tiveram o comprimento aproximadamente duas vezes a sua largura.

- braçadeiras confeccionadas de tecido de brim resistente e fechadas com velcro (material adesivo), permitindo o melhor ajuste em braços cônicos.

- fita métrica.

\subsection{Escolha do manguito de largura correta (MLC)}

O MLC foi escolhido entre as diversas larguras dos manguitos disponíveis, (conjunto pertencente ao Laboratório de Esfigmomanometria-EEUSP), com base na aplicação da equação:

$\mathrm{MLC}=\mathbf{C} \times 1,2$

Onde $\mathrm{C}$ é igual a circunferência do braço em centímetros e 1,2 a constante que permite que o MLC seja $20 \%$ maior que o diâmetro do braço, ou 0,38 de sua circunferência, atendendo-se portanto os princípios preconizados pela AMERICAN HEART ASSOCIATION (1980), com exceção do local de medida da circunferência do braço, no procedimento recomendado por ARCURI (1985), a medida ocorre no ponto mais largo do bíceps braquial e não na metade da distância acrômio-oleacron, como recomendou a associação americana. 


\section{Método utilizado para a coleta de dados}

Com base na lista de cirurgias programadas, a autora convidava os pacientes para integrarem a amostra populacional, explicando-lhes a finalidade da pesquisa, e, diante da sua anuência, iniciava-se o preenchimento do formulário elaborado para coleta de dados (ANEXO I).

Em seguida solicitava-lhes que deitassem e relaxassem os músculos, principalmente o bíceps braquial. Mediu-se a circunferência do braço esquerdo, no ponto mais largo do bíceps braquial, para a escolha do MLC, definindo-a a partir dos valores abaixo:

$\begin{array}{lcc}\begin{array}{c}\text { manguito } \\ \text { circunferência }\end{array} & \begin{array}{c}\text { C } 1,2 \\ (\mathrm{~cm})\end{array} & \begin{array}{c}\text { MLC } \\ (\mathrm{cm})\end{array} \\ 20,21,22 & 7,6 \text { a } 8,4 & 8 \\ 23,24 & 8,5 \text { a } 9,4 & 9 \\ 25,26,27 & 9,5 \text { a } 10,4 & 10 \\ 28,29 & 10,5 \text { a } 11,4 & 11 \\ 30,31,32 & 11,5 \text { a } 12,4 & 12 \\ 33,34 & 12,5 \text { a } 13,4 & 13 \\ 35,36,37 & 13,5 \text { a } 14,4 & 14\end{array}$

Após a escolha do manguito correto, era feita uma medida inicial da pressão arterial para que o participante conhecesse as sensações causadas pela inflação e deflação do manguito, assim como para o autor conhecer os níveis pressóricos. A partir dessa medida inicial, mais 2 medidas eram feitas com MLC e MLP, encerrando-se a primeira fase.

No dia seguinte, iniciava-se a medida da pressão arterial na fase de recepção no centro cirúrgico, onde foram feitas 4 medidas, $2 \mathrm{com}$ MLC e 2 com MLP. Nessa ocasião as medidas foram realizadas na mesma posição do dia anterior, deitada em decúbito dorsal com o braço esquerdo ao nível da croça da aorta formando um ângulo de $45^{\circ}$ em relação à linha axial do tórax e o tempo de espera, para ser encaminhado à sala de operações, não excedia a 30 minutos.

Encerrada essa fase, o paciente era encaminhado à sala de operaraçōes e após o término da cirurgia era transportado à SRA, iniciando-se a fase seguinte (fase pós-anestésica).

As medidas em SRA, foram realizadas durante a permanência do paciente no local, e portanto variaram quantitativamente, tendo em vista que alguns pacientes não necessitaram permanecer na SRA 180 minutos ( 3 horas).

Para análise comparativa das medidas da pressão arterial decidiu-se considerar, em todas as fases, 1 medida com MLC e 1 medida 
com MLP, registradas dentro do intervalo de um minuto, atendendose assim a necessidade da comparação ser efetuada dentro do menor tempo possível.

\subsection{Procedimento padronizado para o registro nas 3 fases}

Nos 101 pacientes estudados o método de obtenção e registro do dado foi padronizado de tal forma, a se garantir o mesmo procedimento em todos os pacientes. Para isso, observou-se os seguintes pontos:

- preparo do paciente para manter-se relaxado durante todo o procedimento, sobretudo durante a troca dos manguitos, deixando-o ciente que após o término da coleta seriam-lhe informados os valores da pressão arterial;

- ajuste perfeito do manguito ao braço, o que foi facilitado pela utilização de velcro ña confecção das braçadeiras.

- garantia de pressão externa uniforme em toda extensão, evitando-se deformação da bolsa de borracha durante a inflação.

- manguito colocado 35 a $30 \mathrm{~mm}$ acima da prega do cotovelo, permitindo colocação do diafragma do estetoscópio na fossa ante cubital.

- inflação de $10 \mathrm{em} 10 \mathrm{mmHg}$ até atingir $30 \mathrm{mmHg}$ acima do valor da pressão arterial sistólica inicial.

- deflação de 2 a $3 \mathrm{mmHg}$ por segundo, aumentando a velocidade para 5 a $6 \mathrm{mmHg}$ nos indivíduos hipertensos evitando desconforto e congestionamento venoso.

- leitura da pressão sistólica no momento do aparecimento dos sons de Korotkoff (fase I) e a diastólica no instante de seu desaparecimento (fase V), segundo as recomendações da AMERICAN HEART ASSOCIATION (KIRKENDALL, 1980).

- garantia de 1 minuto de intervalc entre as medidas feitas com MLC e MLP.

\subsection{Análise dos dados}

As medidas indiretas da pressão arterial com MLC e MLP foram comparadas em cada fase, assim como entre as fases pré-operatoria imediata, de recepção no centro cirúrgico e fase pós-anestésica.

\section{DISCUSSÃO E RESULTADOS}

No estudo dos valores obtidos da pressão arterial de 101 pacientes nas fases pré-operatória imediata, de recepção no centro cirúrgico e pós-anestésica, foram observadas diferenças significativas entre medidas realizadas com MLC e o MLP. De oito comparações realizadas 
nas três fases entre os dois manguitos, em sete, o nível de significância atingiu valores de $\mathrm{p}<0,0001$, com exceção da última medida feita na fase pós anestésica aos 180 minutos.

O manguito mais utilizado foi o de $10 \mathrm{~cm}$ de largura, tanto pela população feminina (28) como pela masculina (19).

Uma vez identificados os manguitos mais utilizados na população estudada, serão apresentados a seguir os valores da pressão arterial obtidos na fase pré-operatória imediata.

TABELA 1 - Medida indireta da pressão arterial sistólica (PAS) e diastólica (PAD) com manguito de largura correta (MLC) e com manguito de largura padrão (MLP) na fase pré-operatória imediata; número de pacientes (N); média (X); desvio padrão (DP); erro padrão (EP); Valor máximo (Vmax); Valor mínimo Vmin). São Paulo, 1988.

\begin{tabular}{|c|c|c|c|c|c|c|c|c|c|c|c|c|}
\hline \multirow[b]{3}{*}{ Manguitos* } & \multicolumn{12}{|c|}{ PRESSAO ARTERIAL } \\
\hline & \multicolumn{6}{|c|}{ SISTOLICA } & \multicolumn{6}{|c|}{ DIASTÓLICA } \\
\hline & $\mathbf{N}$ & $\mathbf{x}$ & $\mathrm{DP}$ & $\mathbf{E P}$ & $V \max$ & $V \min$ & $\mathbf{N}$ & $\mathrm{x}$ & $\mathrm{DP}$ & EP & Vmax & Vmin \\
\hline MLC & 101 & 117,5 & 14,3 & 1,4 & 160,0 & 86,0 & 101 & 78,2 & 12,0 & 1,2 & 100,0 & 54,0 \\
\hline MLP & 101 & 111,2 & 15,0 & 1,5 & 150,0 & 80,0 & 101 & 73,0 & 12,0 & 1,2 & 100,0 & 50,0 \\
\hline
\end{tabular}

* decorrido um minuto entre as medidas

p $<0,05$

Como mostram os dados da tabela 1 existem diferenças significantes entre as médias obtidas pela medida indireta de pressão arterial sistólica feita com MLC e MLP e em alguns pacientes essas diferenças atingiram valores altamente significantes $(p<0,0001)$, destacamos hipoestimações, individuais, de $30 \mathrm{mmHg}$ com o MLP.

Esses fatos, ratificam o questionamento sobre a validade de se utilizar tabelas de correção de erros para resolver o problema imposto pelo MLP (ARCURI, 1986), já que as tabelas são calculadas matematicamente para indicar apenas um valor pré estabelecido para cada valor de circunferência de MLP. preciso considerar que a faixa de variação para braços com circunferências iguais é grande, devido a fatores individuais relevantes, como hereditariedade, tabagismo, componentes emocionais. Tais achados corroboram as observações de IRVINE, (1968) e BERLINER et al (1960) que também constataram relevantes diferenças interindividuais e a dificuldade em se utilizar modelos para correção de erros.

Chama a atenção que embora as diferenças diastólicas entre as medidas obtidas com o MLC e o MLP sejam também significantes $(p \leqslant 0,05)$, não se observou diferenças entre o desvio padrão, erro padrão e valores máximos. $E$ possível que o fato da resposta sistólica ser geralmente mais intensa que a diastólica, diante de estímulos estressantes, possa explicar os resultados obtidos. 
A tabela 2 revela os valores da pressão arterial no momento em que o paciente é recebido no centro cirúrgico.

TABELA 2 - Medida da pressão arterial sistólica (PAS) e diastólica (PAD) com manguito de largura correta (MLC) e manguito de largura padrão (MLP) na fase de recepção no centro cirúrgico: número de pacientes $(N)$; média $(X)$; desvio padrão (DV); erro padrão (EP); Valor máximo (Vmax) Valor mínimo (Vmin). São Paulo, 1988.

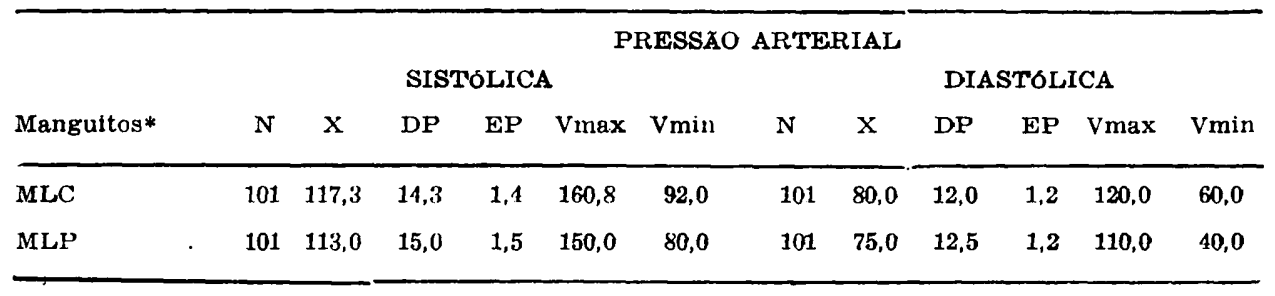

* decorrido um minuto ente as medidas

$\mathbf{p}<\mathbf{0 , 0 5}$

No momento em que o paciente foi recebido no centro cirúrgico as diferenças entre os dois manguitos permaneceram significantes $(p \leqslant 0,05)$, chama atenção certa aproximação dos valores referentes ao desvio padrão e erro padrão, que continuam sendo menores com o MLC.

Considerando os fatores emocionais implicados na fase pré-operatória imediata, poder-se-ia esperar maior labilidade de pressão arterial em alguns indivíduos, tendo em vista o potencial para maior reatividade vascular implicada a aspectos neurais, hemorragias, fatores hormonais e outros mecanismos. $\$$ preciso considerar entretanto que nessa fase os pacientes já se encontram sob efeito das drogas indicadas no pré-anestésico, o que possivelmente poderia auxiliar certos ajustes neurológicos minimizando labilidades de pressão arterial.

Com respeito aos valores máximo e mínimo, entretanto, a tabela 2 indica diferenças entre os manifestantes, mostrando que o aumento da pressão sistólica de alguns indivíduos foi melhor registrada pelo MLC devido provavelmente ao seu melhor ajuste no braço. Esse raciocínio também se aplica às importantes diferenças observadas nas pressões diastólicas, nos valores máximo e mínimo, pois com MLC foi observado aumento de $20 \mathrm{mmHg}$ no valor máximo da fase pré-operatória e de recebimento no Centro Cirúrgico. Resta indagar se a maior reatividade da pressão arterial observada, estaria ligada a fatores como hipertensão, em potencial ou declarada, e ausência de medicação pré-anestésica, fatores esses que talvez pudessem estar implicados nas alterações da pressão arterial. Caso esses fatores pudessem estar excluídos, restaria pensar na possibilidade de um dos pacientes apresentar maior alteração na pressão arterial, diante da situação de medo e suas consequiências.

A tabela 3 apresenta os dados obtidos na fase em que o paciente permaneceu na SRA, após o ato cirúrgico. 
TABELA 3 - Medida indireta da pressão arterial sistólica (PAS) e diastólica (PAD) com manguito de largura correta (MLC) e com manguito de largura pa. drão (MLP) na fase pós-anestésica: número de pacientes (N): média )X); desvio padrão (DP); erro padrão (EP); Valor máximo (Vmax); Valor mínimo (Vmin). São Paulo, 1988.

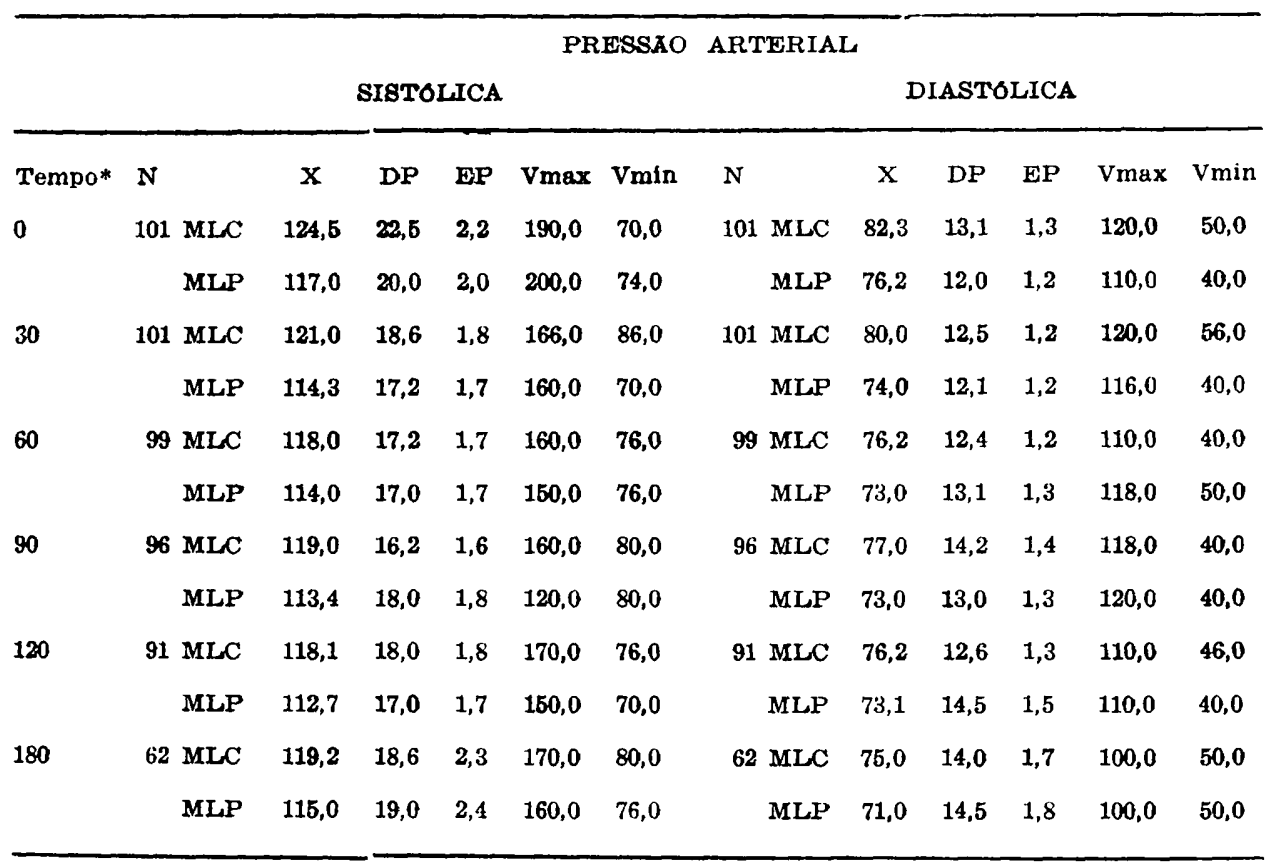

* decorrido um minuto ente as medidas

Como pode ser observada no momento de sua admissão na SRA (tempo 0), importante incremento ocorre nos níveis da pressão arterial com relação à fase de recepção no centro cirúrgico. Embora este estudo não tenha por objetivo discutir os mecanismos implicados nas alterações da pressão, sugere-se que fatores inerentes ao retorno da consciência, dor, drogas administradas, retenção urinária, aumento de débito cardíaco por hipervolemia (infusão venosa de soro), ansiedade e outros, possam influenciar nessas alterações.

Destaca-se que no momento em que os pacientes chegam na SRA, muitas vezes se encontram em estado de semi-consciência, mas já nesse instante, é iniciado sua estimulação para respiração profunda, movimento de membros, teste de força muscular, etc.

$E$ interessante observar, que embora aos 60 minutos os níveis registrados são semelhantes aos do período pré-operatório, após 120 minutos ocorre um discreto aumento dos níveis sistólicos, porém só observado com o uso do MLC. possível que ao recuperar a cons- 
ciência, o aumento do estado de alerta, relacionado à necessidade de retornar à enfermaria, conhecer o resultado da cirurgia, rever os parentes mais próximos e aumento da dor estejam mais relacionados às alterações da pressão sistólica. $\mathrm{E}$ preciso considerar entretanto esse dado, apenas como parâmetro indicativo para estudos futuros, não sendo conclusivo nesta ocasião.

Como ficou patente, o MLP ocasiona hipoestimações significantes em todas as fases estudadas, tornando a avaliação da pressão arterial com MLP vulnerável.

COHN; DADDARIO (1965); MECCA (1983) afirmaram que o baixo fluxo sanguíneo nas situações de choque e a hipotermia são as maio. res causas de imprecisão da medida indireta. VAUGHAN et al (1981) relatam que $60 \%$ dos pacientes que chegam à SRA, estão significantemente hipotérmicos o que desencadeia a liberação de adrenalina causando a vasoconstrição periférica, sendo o período pós-anestésico o momento menos favorável para compensar esse mecanismo.

Qual o significado dessas diferenças? Qual o prejưzo quando além da, hipotermia e baixo fluxo sanguíneo, situações comuns em SRA, o braço é tão fino que não permite o ajuste necessário para completa transmissão da pressão do braço para a câmara de borracha? O erro provocado pelo MLP pode conduzir a administração de drogas desnecessárias em indivíduos magros durante os períodos anestésico cirúrgico e pós anestésico? Essas e outras questões conduzem a reflexão sobre a precisão da medida da pressão, no momento em que a avaliação desse parâmetro assume importância indiscutível, ou seja, nas unidades críticas, como é a SRA, local onde a assistência de enfermagem é a atividade primordial para se detectar e prevenir possiveis complicações e onde o paciente permanece, após ter sido submetido ao trauma cirúrgico, emergindo de um período de insta. bilidades, até atingir sua homeostase.

\section{CONCLUSÓES}

O estudo da medida indireta da pressão arterial em função da largura do manguito, em pacientes nas fases pré-operatória imediata, recepção no centro cirúrgico e pós-anestésico conduziu às seguintes conclusões:

- Na população estudada, foi verificado que o manguito mais utilizado foi o de $10 \mathrm{~cm}$ de largura em ambos os sexos, e $96,9 \%$ dos 101 pacientes estudados necessitaram de largura de manguitos menores que $12 \mathrm{~cm}$.

- As médias obtidas com o MLC nas fases estudadas variaram entre $117,3 \mathrm{mmHg}$ a $124,5 \mathrm{mmHg}$ nos valores sistólicos e $75,0 \mathrm{mmHg}$ a $82,3 \mathrm{mmHg}$ nos valores diastólicos.

- As médias obtidas com MLP nas fases estudadas variaram entre $111,2 \mathrm{mmHg}$ e $117,0 \mathrm{mmHg}$ nos valores sistólicos e $71,0 \mathrm{mmHg}$ e $76,2 \mathrm{mmHg}$ nos valores diastólicos. 
O nivel de significância na comparação entre as médias foi de $\mathrm{p}<0,05$, constatando-se uma dispersão que resultou no nivel de $\mathrm{p}<0,0001 \mathrm{em}$ alguns indivíduos.

As diferenças individuais mais importantes foram encontradas no período pré-operatório onde a média sistólica foi hipoestimada pelo MLP em cerca de $6,3 \mathrm{mmHg}$ e a diastólica em $5,2 \mathrm{mmHg}$ tendo-se atingido valores individuais de até $30 \mathrm{mmHg}$.

PENICHE, A. de C.G.; ARCURI, E.A.M. Measure of blood pressure in function of sizes of cuffs, in patients in mediate perioperative phase, reception area of operation room and posoperative phase. Rev. Esc. Enf. USP, v. 26, n. 2, p. 243-56, Aug. 1992.

The goal of this study was to compare blood pressure measures using two sizes of cuffs: one standard width $(12 \mathrm{~cm})$ and other with correct width that is 0,38 of arm circumference as recommended by American Heart Association. The comparisons were done among surgical patients in mediate perioperative phase, reception area of operation room and postoperative phase during the staying of the patient in the recovery room. The results demonstrated that the arterial blood pressure was hipoestimated by the use of the standard width cuff, reaching values up $30 \mathrm{mmHg}$ in the systolic arterial pressure and $30 \mathrm{mmHg}$ in the diastolic ones.

UNITERMS: Blood pressure. Blood pressure determination.

\section{REFERENCIAS BIBLIOGRÁFICAS}

1. ALDRETE, J.A.; KROULIK, D. A postanesthetic recovery score. Curr. Hes. Anesth., v. 49, n. 6, p. $924-33,1970$.

2. AMERICAN HEART ASSOCIATION. Recommendations for human blood pressure detorminations by sphygmomanometers. Dallas, 1980.

3. AMERICAN HEART ASSOCIATION AND THE CARDIAC SOCIETY OF GREAT BRITAIN AND IRELAND. Standardization of blood pressure readin:ss. Am. Heart J., v. 18, p. 95101, 1939. Supplement.

4. ARCURI, E.A.M. et al. Estudo comparativo da medida indireta da pressão arterial cum manguito de largura correta e com manguito de largura padrão. Arq. Bras. Cardiol., v. 47, n. 2, p. 143-4, 1986.

5. ARCURI, E.A.M. Medida da pressão arterial pelo método auscultatório: necessidade de utilizaç̃o de manguito de largura adequada para detecção precoce da hipertensão. Săo Paulo, 1988. 91 p. Tese (livre docência) - Escola de Enfermagem, Universidade de São Paulo.

6. BAZET, H.C.; LAPLACE, L.B. Studies on the indirect measurement of blood pressure. I sources of error in the Riva Rocci Method. Am J. Physiol., v. 103, n. 1, p. 48-67, 1933.

7. BERLINER, $K$. et al. The accuracy of blood pressure determinations: a comparison of direct and indirect measurements. Cardiologia, v. 37, n. 1, p. 118-28, 1960.

8. CASTELLANOS, B.E.P.; JOUClas, V.M.G. Assistência de enfermagem perioperatória Um modelo conceitual. Rev. Esc. Enf. USP, v. 24, n. 3, p. 359-69, 1990.

9. COHN, J.N.; DADDARIO, R.C. Mechanisms of disappearance of Korotkotf sounds in clinical shock. Circulation, v. 31/32, p. 69, 1965. Supplement. 2.

10. CONSEILLER, C. et al. Complications des 24 premiers heures post operatoires. Paris, Librairie Arnette, 1975.

11. CULLEN, J.D. Recovery room complications. AORN. J., v. 26, n. 4, p. 746-63, 1977. 
12. CUNHA, A.M.P. et al. Análise de rotina de verificação dos sinais vitais dos pacientes pós-operatório imediato, em um centro de recuperação anestésica. Enf. Novas vimens., v. 3, n. 2, p. $11420,1977$.

13. DRAIN, C.B.; SHIPLEY, B.S. Enfermagem na sala de recuperacão. Rio de Janeiro, Interamericana, 1981.

14. FERRAZ, E.R. Requisitos minimos para a organização da sala de recuperação pós-anestésica e a assistência de enfermagem nela prestada. Rov. Esc. Wnf. USP, v. 14, n. 2, p. 123-31, 1980 .

15. IRVINE, R.O.H. The influence of arm girth and cuff size on the measurement of blood pressure. N. Z. Med. J., v. 67, 279-83, 1968. Supplement.

16. JOUCLAS, V.M.G. Análige da funcão do circulante de sala de operacões de acordo com a metodologia sistêmica de orgunização de recursos humanos. Săo Paulo, 1987. 313 p. Tese (Dutorado) - Escula de Enfermagem, Universidade de São Paulo.

17. KARVONEN, M.J. et al. Sphygmomanometer cuff size and accurancy of indirect measurement of blood pressure. Am J. Cardiol., v. 13, p. 688-93, 1964.

18. KIRKENDALL, W.M. et al. Recummendations for human blood pressure determination by sphygmomanometers. Circulation, v. 62, 11. 5, p. 1146-55, 1980.

19. MANCIA, G. et al. Alerting reaction and rise in blood pressure during measurements by physician and nurse. Hypertension, v. 9, n. 2, p. 209-15, 1987.

20. MECCA, R.S. Hypotension in the recovery room. Curr. Rev. Rocov Room Nurses, v. 5, n. 3, p. 18-24, 1983.

21. NOCITE, J.R. Recuperação pós anestésica: aspectus geraís. Bev. Bras. Anestesiol., v. 37, n. 3, p. 161-7, 1987.

22. I'OMIDOSSI, G. et al. Absence of alarm reactions with use of non invasive bloud pressure monitoring devices. Clin. Exp. Hypertens., (A), v. 7, n. 2/3, p. 429-36, 1985.

23. FOSSO, M.B.S. Avaliação das condiçōes dos pacientes nas salas de recuperaçũo pós-aneytésica. Rev. Esc. Enf. USP, v. 9, n. 3, p. 9-23, 1975.

24. POUND, J.L. Consistency in measurement of blood pressure in the postanesthesia care unit. J. Post. Anesth. Nurs, v. 2, n. 2, p. 78-83, 1987.

25. KOSE, G.A. et al. A sphygmanometer for epidemiologists. Lancet, v. 1, p. 614-18, 1966.

26. VAUGHAN, M.S, et al. Postoperative hypothermia in adults: relationship of age, anesthesila and shivering to rewarning. Anesth Analg, v. 60, n. 10, p. 746-51, 1981.

27. WEBB, G.E. Hyper and hypotension in the recovery room. AORN J., v. 26, n. 3, p. 546$74,1977$.

28. WILCOX, J. Observer factors in the measurement of blood pressure. Nurs, Res., v. 10, n. 1, p. 4-17, 1961 . 


\section{ANEXOS}

NOME: $N$

Idade: Sexo: Cor:

Medicação pré-anestésica: sim กฉ̃o

Circunferência do braço:

Manguito utilizado

Pressão arterial na fase pré-operatória imediata

Pressão arterial inicial: $\mathrm{X}$

1\% MLC $\mathrm{X}$. ; 2 a $\mathrm{MLC}$ $\mathbf{X}$

3. MILP $\mathrm{X}$ ; $4^{\mathrm{a}}$ MLP $\mathrm{X}$

Pressão arterial na fase de recepção no centro cirúrgico

1. MIC $\mathbf{X}$ ; $2^{\mathrm{a}} \mathrm{MLC}$ $\mathbf{x}$

3* MIP $\mathbf{X}$ ; $4^{2} \mathrm{MLP}$ $\mathbf{x}$

\section{Pressão arterial na fase pós anestésica}

$(t=0$ minuto $)$

19. MLC $\mathbf{x}$ ; $2^{\mathrm{a}} \mathrm{MLC}$ $\mathbf{x}$

39. MUP $\mathrm{X}$ ; 4. MLP $\mathrm{x}$

$(t=30$ minutos $)$

1. MLC___ $\mathrm{X}$

3. MILP $\mathbf{X}$ ; 2a MLC $\mathrm{X}$

$(t=60$ minutos $)$

1\% $\mathrm{MLC}$ ; 2 a MLC X

3a MLP $\mathrm{X}$ ; 4* MLP $\mathrm{X}$

$(t=90$ minutos $)$

1: MLC ; 2* MLC $\mathbf{x}$

3a MIP ; 4 MLP X.

$(t=120$ minutos $)$

1. $\mathrm{MLC}$ ; 2 MLC X.

3a MLP X. ; 4. MrP $\mathrm{X}$

$(t=180$ minutos $)$

1. $\mathrm{MLC}$ $\mathbf{X}$ ; 2. MLC $\mathbf{x}$

3a MIP $\mathrm{x}$ ; 4 MLP $\mathrm{X}$ 